\title{
Predictors of exercise capacity in patients with atrial correction of transposition of great arteries
}

\author{
Antonova $\mathrm{P}^{1}$, Chaloupecky $\mathrm{V}^{3}$, Adla $\mathrm{T}^{2}$, Tomek V , Slaby $\mathrm{K}^{4}$, Zeman $\mathrm{J}^{5}$, Rohn $\mathrm{V}^{1}$ \\ Center for Adult Congenital Heart Disease, Cardiovascular Surgery Clinic, 2nd Medical Faculty, Charles University \\ in Prague and Motol University Hospital, Praha, Czech Republic. petra.antonova@fnmotol.cz
}

\section{ABSTRACT}

OBJECTIVES: To examine the relationship of clinical, biochemical and imaging parameters to maximum oxygen uptake in patients after atrial correction of transposition of great arteries.

BACKGROUND: Exercise tolerance is a key determinant of quality of life in patients with adult congenital heart disease. It is determined by a large scale of factors often different from general cardiology.

METHODS: 86 consecutive patients after Senning correction of TGA were subjected to clinical and echocardiographic examination, Holter monitoring, blood tests of NT-proBNP, MRI of the heart and exercise test. Parameters of these examinations were correlated to $\mathrm{VO}_{2}$ max.

RESULTS: The average age of patients was $28 \pm 3.5$ years. The average systemic right ventricular function determined by MRI was $51.9 \pm 7.9 \%$. The average NT-proBPN was $124.3 \pm 23.6 \mathrm{ng} / \mathrm{l}, \mathrm{VO} \mathrm{2}_{2} \max .31 .7 \pm 6.5$ $\mathrm{ml} / \mathrm{kg} / \mathrm{min}$ and the heart rate reserve $106 \pm 24 / \mathrm{min}$. Neither systemic right ventricular systolic function nor NT-proBPN predicted $\mathrm{VO}_{2}$ max., whereas the heart rate reserve did $(\mathrm{p}=0.003)$.

CONCLUSION: An inability to increase heart rate during exercise noted in a considerable number of patients after atrial switch of TGA caused a decreased exercise tolerance. It is not solely the global systolic function of either ventricle that influences the exercise performance, rather it is the ability to increase heart rate and overall cardiac output appropriately (Tab. 3, Fig. 6, Ref. 28). Text in PDF www.elis.sk KEY WORDS: transposition of great arteries, Senning correction, maximal oxygen uptake, right ventricular systolic function, heart rate reserve.

\section{Introduction}

It is generally known that patients after Senning (atrial) correction of transposition of great arteries (TGA) survive well into adulthood. The expected long-term mortality is acceptable, with the survival rates reaching $75 \%$ at 30 years follow up $(1,2,3)$. Patients' morbidity and quality of life are determined largely by an exercise tolerance (4). There is an interest in investigating systemic right ventricular systolic function (RVEF) and its influence on exercise and functional capacity $(5,6,7,8,22)$. The attention also focuses on other pathophysiologic mechanisms influencing

${ }^{1}$ Center for Adult Congenital Heart Disease, Cardiovascular Surgery Clinic, 2nd Medical Faculty, Charles University in Prague and Motol University Hospital, Praha, Czech Republic, ${ }^{2}$ Department of Imaging Methods, 2nd Medical Faculty, Charles University in Prague and Motol University Hospital Praha, Czech Republic, ${ }^{3}$ Children's Heart Centre, 2nd Medical Faculty, Charles University in Prague and Motol University Hospital, Praha, Czech Republic, ${ }^{4}$ Sports Medicine, 2nd medical Faculty of Medicine, Charles University in Prague and Motol University Hospital, Praha, Czech Republic, and ${ }^{5} 3$ rd Medical Faculty, Charles University Prague, Královské Vinohrady Hospital, Praha, Czech Republic

Address for correspondence: P. Antonova, Center for Adults with Congenital Heart Disease, Cardiovascular Surgery Clinic, University Hospital Motol, V Úvalu 84, CZ-1590 00 Praha 5, Czech Republic. Phone: +420224435250, Fax: +420224435220, email:

Acknowledgement: Supported by the Project of Internal Grant Agency of Ministry of Health, Czech Republic (NT 13408-4/2012). exercise tolerance such as: atrial baffles capacitance and diastolic function of both ventricles $(9,10)$. Potential predictors of low functional capacity and cardiac insufficiency as B-type natriuretic peptide (NT-proBNP) and other markers have been investigated. Nevertheless, none of above the mentioned parameters showed a consistent relationship to exercise tolerance throughout available literature data. Our aim was to evaluate a set of clinical, biochemical and imaging parameters that can be easily accessible in daily clinical practice and examine their association with the maximal oxygen uptake $\left(\mathrm{VO}_{2}\right.$ max.), when evaluating the exercise capacity.

\section{Materials and methods}

86 consecutive patients after Senning correction of TGA in childhood, presenting for a regular examination in the outpatient service of the Center for Adults with Congenital Heart Disease at the University Hospital Motol in Prague within the period of one year, were subjected to clinical and echocardiographic examination, Holter monitoring, blood tests of NT-proBNP, MRI of the heart and the exercise test. Their median age at operation was 0.62 years and the median age at follow-up examination was 27.2 years. Patients with permanent cardiac pacemakers were excluded from the study due to the inability to undertake cardiac MRI. The study was approved by the hospital ethical committee and all the patients signed an informed consent. 
Tab. 1. Patient demographic and clinical characteristics.

\begin{tabular}{ll}
\hline Female sex, n (\%) & $22(25.6 \%)$ \\
\hline Age at operation, y, mean (SD) & $1.42(0.86)$ \\
Age at operation, y, median & 0.62 \\
Age at clinical examination, y, mean (SD) & $28(3.5)$ \\
Age at clinical examination, y, median & 27.2 \\
NYHA class, mean (SD) & $1.3(0.44)$ \\
NT-proBNP (ng/l), mean (SD) & $124.3(23.59)$ \\
\hline
\end{tabular}

\section{Clinical examination and diagnostic tests}

A detailed history focused on functional status and the quality of life. New York Hospital Association functional class was recorded. A thorough questioning and examination for signs and symptoms of cardiac insufficiency was performed. All medications were noted. Blood tests for NT-proBNP were drawn. Standard Holter ECG 24- hour monitoring was performed and the presence of disorder of sinus node function presented as either junctional rhythm or ectopic atrial rhythm was recorded.

\section{Echocardiography}

Echocardiography was performed on Vivid 9 analyser (GE Medical Health Systems) with 2.5-5 MHz convex transducers by one experienced examiner. Transthoracic examinations were performed in supine position using standard 2D grayscale and Doppler techniques. All measurements were performed in standard projections in compliance with American Society of Echocardiography guidelines. Right ventricular systolic function was assessed semi-quantitatively and characterized as good, when the estimated ejection fraction was above $50 \%$, moderately impaired (35-50 \%) and severely impaired (less than $35 \%$ ). Systemic tricuspid valve regurgitation was graded as mild, moderate or severe.

\section{MRI}

All examinations were performed on 1,5T MRI Avanto equipment. Standard protocol included static steady-state free procession (SSFP) in axial, coronal and sagittal planes, cine SSFP and contrast enhanced angiography. For volume measurements, consecutive cine SSFP retrospectively gated images were used (slice thickness $8 \mathrm{~mm}$, gap $2 \mathrm{~mm}, 25$ phases). Manual delineation of the endocardial borders was performed in end-diastole and end-systole. Ejection fraction (EF) was calculated as (End Diastolic VolumeEnd Systolic Volume/ End Diastolic Volume) and expressed in percent for both systemic right and sub-pulmonary left ventricles.

\section{Exercise testing}

Exercise testing was conducted on electromagnetically braked bicycle ergometer (Ergoselect 100P, Ergoline, Bitz, Germany).

Tab. 2. Echocardiographic and MRI parameters of systemic right ventricle systolic function.

\begin{tabular}{lccc}
\hline & Good, $\mathrm{n}(\%)$ & Moderately impaired, $\mathrm{n}(\%)$ & Severely impaired, $\mathrm{n}(\%)$ \\
\hline Echocardiography & $52(60)$ & $31(36)$ & $3(3.4)$ \\
MRI & $48(55,8)$ & $36(36)$ & $2(2.3)$ \\
\hline
\end{tabular}

The right ventricular systolic function was assessed semi-quantitatively by echocardiography and characterized as good, when the estimated ejection fraction was above $50 \%$, moderately impaired $(35-50 \%)$ and severely impaired (less than $35 \%$ ). The same criteria were used for MRI assessment of right ventricular systolic function.
Tab. 3. Correlation coefficients for $\mathrm{VO}_{2} \max$ and given parameters.

\begin{tabular}{lcc}
\hline Parameter & $\rho$ value & p value \\
\hline NT-pro BNP & 0.0706 & 0.608 \\
NYHA & & 0.037 \\
RVEF echo & 0.029 \\
RVEF MR & 0.0752 & 0.502 \\
LVEF MR & 0.1295 & 0.282 \\
HRR & 0.3247 & 0.003 \\
\hline
\end{tabular}

RVEF - right ventricular ejection fraction; LVEF - left ventricular ejection fraction; MR - magnetic resonance; HRR - heart rate reserve

Standardised protocol with 0.5 Watt/kilogram increments every three minutes was performed. Respiratory gas analysis of the breath was performed using a properly calibrated gas analyser Oxycon Pro with electrochemical oxygen sensor (CareFusion, Hoechberg, Germany). Heart rate and ECG were monitored continuously. At each step of exercise, peripheral capillary oxygen saturation, heart rate, blood pressure and ECG were registered. Exercise was terminated at the limit of patients staying power, but patients were strongly encouraged to continue with exercise till there was an evidence of anaerobic metabolism. None of the patients had to terminate exercise due to chest pain, arrythmia or descent of systolic blood pressure. The maximal workload relative to body weight, maximal oxygen uptake, and heart rate reserve were recorded. Heart rate reserve (HRR) was calculated as the heart rate at peak exercise minus resting heart rate.

\section{Statistical analysis}

Continuous data are reported as the means \pm SD and medians and the range and categorical variables as counts and percentages. Student's tests were performed for paired comparisons, when data was normally distributed. The analysis of relations among the studied variables was performed using non-parametric statistical methods such as: Kruskal-Wallis ANOVA, Mann-Whitney test, Fisher exact test and Spearman correlation coefficient. Analysis was performed in R statistical software, version 3.4.2, R Core Team (2017). p-values less than $5 \%$ were considered statistically significant (11).

\section{Results}

The average age of the patients at examination was $28 \pm 3.5$ years, their median age was 27.2 years and $74 \%$ were men. NYHA I class was reported by 63 (73\%) of patients, NYHA II by $22(26 \%)$ and only one patient stated NYHA class III (Tab. 1). Holter monitoring detected episodes of junctional or ectopic atrial rhythm in $45 \%$ of patients and none atrioventricular block. Right ventricular function determined by echocardiography was normal in $52(60.1 \%)$ patients, moderately decreased in 31 (36.5\%) and only in three $(3.4 \%)$ patients it was classified as severely decreased. MRI diagnosed 2 patients $(2.2 \%)$ with a severely decreased right ventricular function, $36(36 \%)$ with a moderately decreased function and in $48(55.8 \%)$ patients, the right ventricular function was 


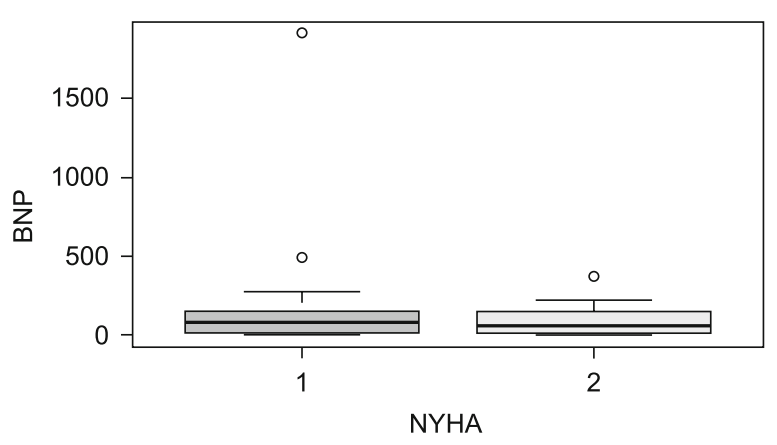

Fig. 1. Relation of NYHA to NT-proBNP.

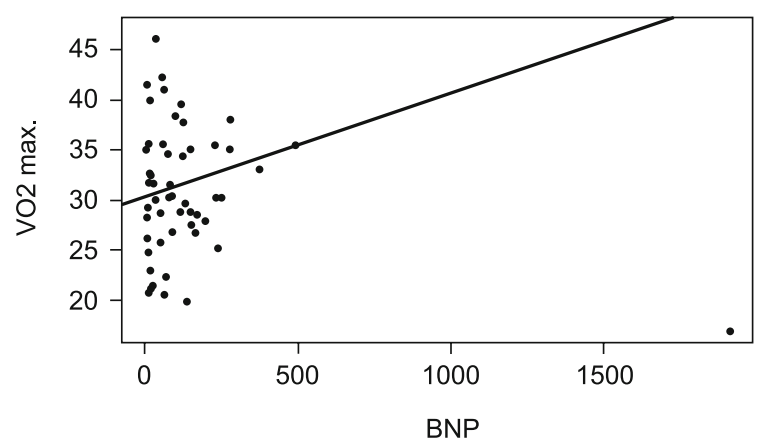

Fig. 2. Relation of NT-proBNP to $\mathrm{VO}_{2}$ max.

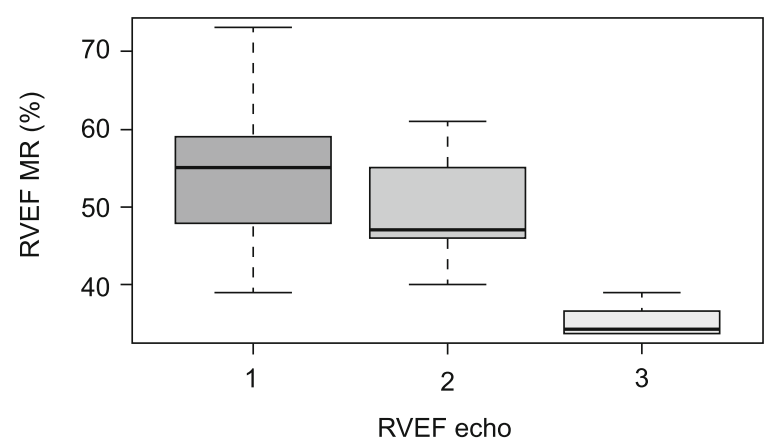

Fig. 3. Comparison of systemic right ventricular function assessed by echocardiography and MRI.

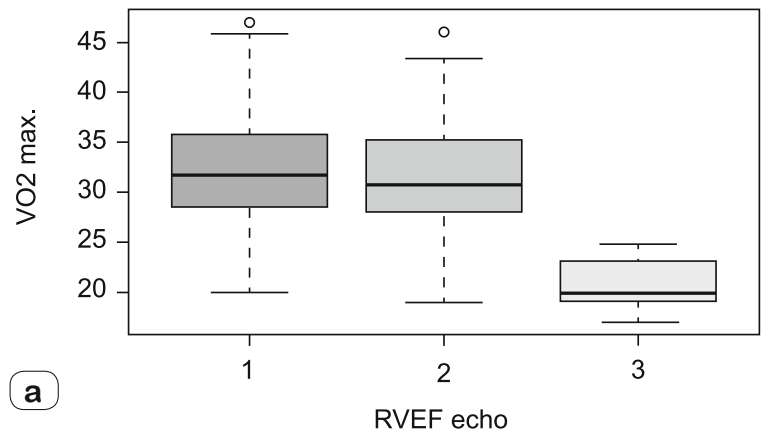

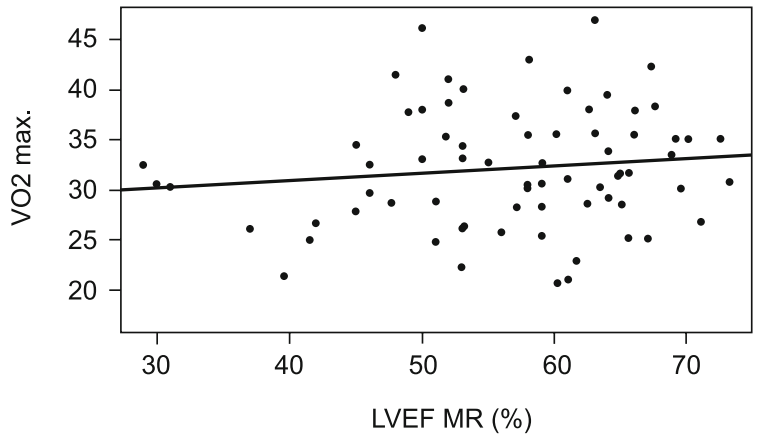

Fig. 5. Relation of MRI LVEF to $\mathrm{VO}_{2}$ max.

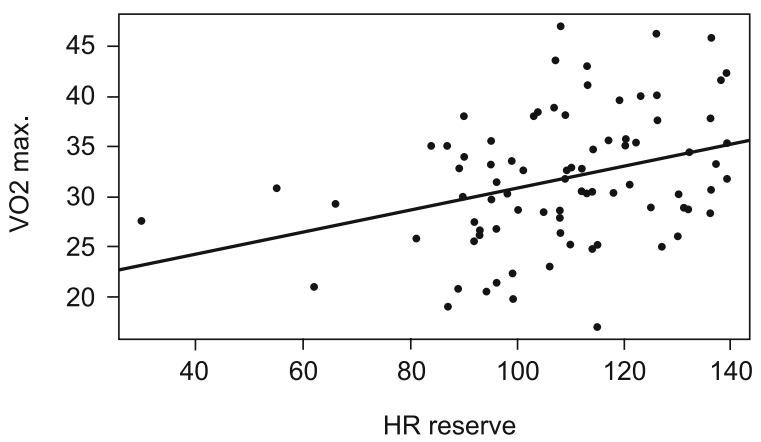

Fig. 6. Relation of heart rate reserve (HRR) to $\mathrm{VO}_{2}$ max.

normal (Tab. 2). The mean systemic right ventricular function assessed by MRI was $51.9 \pm 7.9 \%$, whereas the mean subpulmonal left ventricular function was $56.7 \pm 9.9 \%$. The mean NT-proBNP was $124.3 \pm 23.59 \mathrm{ng} / \mathrm{l}$, elevation of this marker above $450 \mathrm{ng} / \mathrm{l}$ was present only in two patients and just one patient already listed for heart transplantation presented with NT-proBNP value above $1000 \mathrm{ng} / \mathrm{l}$. The mean $\mathrm{VO}_{2}$ max during exercise was $31.7 \pm 6.5$ $\mathrm{ml} / \mathrm{kg} / \mathrm{min} .12$ patients were found to be in the range below 1 standard deviation (SD) and one ranked below 2 SDs range. The mean heart rate reserve was $106 \pm 24 / \mathrm{min}$.

NT-proBNP did not predict neither NYHA class $(\mathrm{p}=0.81)$ nor $\mathrm{VO}_{2} \max (\rho=0.0706, p=0.60)$ (Figs 1 and 2$)$. The ejection fraction of the systemic right ventricle estimated by echocardiography cor-

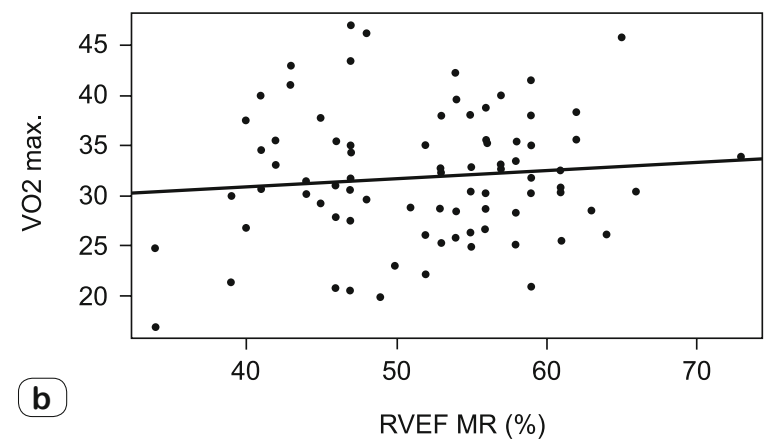

Fig. 4 a, b. Relation of echocardiographic and MRI RVEF to $\mathrm{VO}_{2}$ max. 
related well with the one calculated by MRI (Fig. 3). Echocardiography however tended to slightly overestimate EF. (Comparison of groups (Kruskal-Wallis test): $\mathrm{p}=0.001$ Test of independence (Fisher's exact test): $\mathrm{p}=0.037)$ ).

The right ventricular function assessed by echocardiography in semiquantitative mode predicted $\mathrm{VO}_{2} \max$ (Comparison of groups (Kruskal-Wallis test): $\mathrm{p}=0.03)$ ), whereas the ejection fraction in percentage described by MRI did not correlate with $\mathrm{VO}_{2}$ max. (Spearman's correlation coefficient: $\rho=0.07, p=0.502$ ) (Figs 4 $\mathrm{a}, \mathrm{b})$. The ejection fraction of the left subpulmonary ventricle did not correlate with $\mathrm{VO}_{2}$ max either (Spearman's correlation coefficient: $\rho=0.13, p=0.28$ ) (Fig. 5). The heart rate reserve was the only parameter that statistically correlated with $\mathrm{VO}_{2}$ max. (Spearman's correlation coefficient: $\rho=0.32, p=0.003$ ) (Fig. 6).

\section{Discussion}

Maximal oxygen uptake is perceived to be a core parameter of exercise tolerance and capacity. It predicts survival in patients with a systemic left ventricular dysfunction (12). The same relationship has not been proven for the right ventricle in the systemic position. However, the exercise capacity and functional class are among the most important predictors of the quality of life of patients with congenital heart disease including TGA after atrial correction (13). We sought to examine, which of routinely used fundamental clinical, biochemical and diagnostic parameters in the patients with systemic right ventricle including NYHA class, NT-proBNP, echocardiographic a MRI derived RVEF and HRR would be statistically associated with $\mathrm{VO}_{2}$ max. Our one-year study comprised the follow up of all consecutive patients after Senning correction of TGA. Patients with permanent pacemakers did not participate in the study as they could not had undergone MR examination of the heart.

It was not surprising that no correlation of NYHA class to $\mathrm{VO}_{2}$ max was observed. NYHA is a subjective measure of shortness of breath and fatigue, when performing activity. Our finding may reflect CHD patients' habitual limitation of being acclimated to low exercise tolerance, which they do not perceive as unusual. Lifelong living with CHD downgrades a person's expectation as to what "normal activity" means. Also, a restriction of physical activity by parents, care-takers and teachers plays an important role as a possible confounder, when assessing NYHA class by patients. Unfortunately, the same is valid for the ability index sometimes used as an alternative of NYHA, when assessing the functional capacity in Adult Congenital Heart Disease population (14).

NT-proBNP is a known predictor of short and long- term mortality in TGA (15) and in other congenital heart diseases as well (23). Unexpectedly, we found that the average and median values of NT-proBNP in TGA population were heterogenous throughout the published literature. Our observed NT-proBNP values were low, when compared to Popelova et al (15), who reported the mean value of $203 \mathrm{ng} / \mathrm{l}$ and were higher than those reported by Plymen et al (6), where the mean NT-proBNP was $40 \pm 35 \mathrm{ng} / \mathrm{l}$. Indeed, the span of values stated in literature for the same age group of TGA atrial switch patients was surprisingly broad. The number of NT-
proBNP values exceeding the generally accepted threshold for cardiac insufficiency in our study was low. Finally, NT-proBNP in our study did not correlate with $\mathrm{VO}_{2}$ max.

The ejection fraction of systemic right ventricle is generally assumed to be the fundamental parameter determining the exercise capacity in TGA after an atrial switch - analogous to the left ventricle in patients with a conventional left sided heart failure. We assessed the systolic function semi-quantitatively by echocardiography and more precisely by MRI computation. Both measurements correlated well one to another despite echocardiography tending to slightly overestimate the EF. The systemic right ventricular function in our study assessed by MRI was good in concordance with the findings reported by other investigators Plymen et al (6) $-51.4 \pm 7 \%$, LaRocca et al (7) $-56 \pm 7 \%$ and Lorenz et al (16) $-51 \pm 9 \%$.

Overall, the systemic RV function assessed semi-quantitatively by echocardiography correlated well with $\mathrm{VO}_{2}$ max, while measurements provided by cardiac MRI did not. This finding is in concordance with Helsen et al (5), Samarai (26) and LaRocca et al (7), where RVEF did not predict $\mathrm{VO}_{2}$ max either. Nonetheless, it is in contrary to Gavotto (28) and Buys et al (17), where ,type of surgery, total energy expenditure and right ventricular contractility were independent predictors of peak $\mathrm{VO}_{2}$ “. Our ambivalent findings thus contribute to the large amount of controversial literature data attempting to investigate hypothesis, whether the systemic right ventricular systolic function determines the maximal oxygen uptake. The final verdict still remains to be pronounced. Possibly, a more complex echocardiographic assessment including longitudinal function parameters, ie. global longitudinal strain and $3 \mathrm{D}$ volumetric function parameters should be employed into comprehensive evaluation of the patients with the systemic right ventricle (27).

Systolic left ventricular function was normal in our cohort and also in other reports in literature $(7,16)$. As the cardiac output of systemic right ventricle $(2.5 \pm 0.8 \mathrm{ml})$ and left ventricle end diastolic volume (LVEDV) were significantly lower than in the control group in Buys et al (17), we can speculate that filling patterns and atrial baffle capacitance (primary determinants of diastolic filling) and preload might also play an important role in influencing cardiac output and overall exercise capacity as reported by Reich and others $(10,18,9)$.

The most striking finding emerging from our study was that exercise capacity of our patients compared to scores reported in literature was higher than in other cohorts - the study from Inuzuka et al (21) mentioned the average $\mathrm{VO}_{2}$ max. $24.2 \pm 7.5 \mathrm{ml} / \mathrm{min} / \mathrm{kg}$ at age 26.5 years. LaRocca et al (7) found $\mathrm{VO}_{2}$ max. of $23 \pm 4$ $\mathrm{ml} / \mathrm{min} / \mathrm{kg}$ and Li reported $27.8 \pm 7.6 \mathrm{ml} / \mathrm{min} / \mathrm{kg}$ in their cohorts. Our reported $\mathrm{VO}_{2}$ max. is by $32 \%$ higher. The average normal values for the same age group in a normal healthy population were $42-46 \mathrm{ml} / \mathrm{min} / \mathrm{kg}$ in men and $38-41 \mathrm{ml} / \mathrm{min} / \mathrm{kg}$ in women. Our patients reached $77.3 \%$ of these normal values compared to $66 \%$ reported by Inuzuka et al (21) and $64 \%$ by Buys et al (17).

It is well-known that the sinus node disorder is inherent to the status post atrial correction and its prevalence ranges in the published literature from $27 \%$ (19) to $52.8 \%$ (20). During Holter 
ECG monitoring, we detected that $45 \%$ of our patients had the sinus node disorder presenting either as episodes of atrial or junctional rhythm with ensuing inability to increase heart rate adequately during a physical load. It is a logical assumption that inability to appropriately increase heart rate influences an overall exercise capacity. Heart rate reserve was a predictor of $\mathrm{VO}_{2} \max$ in our study. Based on results of our study and our practical experience with the patients with an incorrect pacemaker settings who can - only after a correct adjustment - increase their heart rate during the exercise testing appropriately and only then does their exercise tolerance improve, we propose that inability to increase heart rate is a limiting factor of exercise tolerance. This suggests that not just the stroke volume, but overall heart output influences the exercise capacity.

\section{Conclusion}

The long-term results of the follow up of the patients after Senning correction of TGA were good. More than a half of the patients in our study presented with a systemic right ventricle systolic function in the normal range, while a severe impairment was noted in a small number of patients. The ejection fraction of the systemic right ventricle did not correlate with $\mathrm{VO}_{2}$ max. The mechanism of a decreased exercise capacity in the patients after an atrial switch is probably complex comprising not only the systolic function of right ventricle, but also intricate haemodynamics of a limited flow through atrial baffles and ensuing a diastolic dysfunction of both ventricles. We were able to demonstrate that a chronotropic incompetence presenting as inability to increase the heart rate during the exercise noted in a considerable number of patients after an atrial switch of TGA was one of the causes of a decreased exercise tolerance. Based on our work, we conclude that it is not only systolic function of either ventricle that influences the exercise performance, rather it is the ability to increase the heart rate and overall cardiac output appropriately.

\section{Limitations}

Patients with permanent pacemakers were excluded from the study due to inability to undergo MRI of the heart. 2 patients listed for heart transplantation followed-up at a different cardiology centre were not included in the study. Only patients coming for the regular outpatient follow up were included. This could have influenced the results of the study by narrowing the range of NTproBNP values and $\mathrm{VO}_{2}$ max noted in our cohort by unintentionally excluding patients, who would not had come for regular visit due to medical disability.

\section{Learning points}

Exercise tolerance is a key determinant of the quality of life in adult congenital heart disease patients.

The systemic right ventricular ejection fraction remains moderately decreased in a majority TGA patients after Senning correction.
The systolic function of the systemic right ventricle does not predict the maximum oxygen uptake.

An inability to increase the heart rate appropriately during an exercise is the limiting factor of the exercise tolerance in TGA patients after an atrial correction.

\section{References}

1. Moons $\mathbf{P}$, Gewillig $\mathbf{M}$, Sluysmans $\mathbf{T}$ et al. Long term outcome up to 30 years after the Mustard or Senning operation: a nationwide multicentre study in Belgium. Heart 2004; 90 (3): 307-313.

2. Williams WG, McCrindle BW, Ashburn DA, Jonas RA, Mavroudis C, Blackstone EH. Outcomes of 829 neonates with complete transposition of the great arteries 12-17 years after repair. Eur J Cardiothorac Surg 2003; 24 (1): 1-9.

3. Vejlstrup N, Sorensen K, Mattson E et al. Long-Term Outcome of Mustard/Senning Correction for Transposition of the Great Arteries in Sweden and Denmark. Circulation 2015: 132 (8): 633-638.

4. Gorler H, Onoa M, Thies A et al. Long-term morbidity and quality of life after surgical repair of transposition of the great arteries: atrial versus arterial switch operation. Interact Cardiovasc Thorac Surg 2011; 12 (4): 569-574.

5. Helsen F, De Meester P, Van De Bruaene A et al. Right ventricular systolic dysfunction at rest is not related to decreased exercise capacity in patients with a systemic right ventricle. Int J Cardiol. 2018; 260 (6): 66-71.

6. Plymen C, Hughes M, Taylor AM et al. Systemic Right Ventricular Function late after Atrial Switch Redirection Surgery for Transpostition of the Great Arteries Relates to NT-pro Brain Natiruretic Peptide and Electrocardiographic Parameters, Abstract 1977. Circulation 2009; 120: S581.

7. LaRocca G, Prahash A, Prinz BF. MRI Predictors of Exercise Capacity in Adult Patients after Atrial Correction for Transposition of the Great Arteries, Abstract 3419. Circulation 2006; 114: II_727-II_728.

8. Li W, Hornung TS, Francis DP. Relation of Biventricular Function Quantified by Stress Echocardiography to Cardiopulmonary Exercise Capacity in Adults with Mustard (Atrial Switch) Procedure for Transposition of the Great Arteries. Circulation 2004; 110 (11): 1380-1386.

9. Fratz S' Hager A, Busch R, Kaemmerer H, Schwaiger M, Lange R, Hess J, Stern HC. Patients after atrial switch operation for transposition of the great arteries cannot increase stroke volume under dobutamine stress as opposed to patients with congenitally corrected transposition. Circ J. 2008; 72 (7): 1130-1135.

10. Reich O, Vorísková M, Ruth C, Krejcír M, Marek J, Skovránek J, Hucín B, Samánek M. Long-term ventricular performance after intra-atrial correction of transposition: left ventricular filling is the major limitation. Heart 1997; 78 (4): 376-381.

11. R Core Team (2017). R: A language and environment for statistical computing. R Foundation for Statistical Computing, Vienna, Austria, www.R-project.org.

12. Moons P, Van Deyk K, De Geest S, Gewillig M, Budts W. Is the severity of congenital heart disease associated with the quality of life and perceived health of adult patients? Heart 2005; 91 (9): 1193-1198.

13. Harber MP, Kaminsky LA, Arena R et al. Impact of Cardiorespiratory Fitness on All-Cause and Disease-Specific Mortality: Advances Since 2009. Prog Cardiovasc Dis 2017; 60 (1): 11-20. 


\section{$527-532$}

14. Overgaard D, Schrader AM, Lisby KH, King C, Christensen RF, Jensen HF, Moons P. Explanatory value of the Ability Index as assessed by cardiologists and patients with congenital heart disease. Congenit Heart Dis 2012; 7 (6): $559-564$.

15. Popelová JR, Tomková M, Tomek J. NT-proBNP predicts mortality in adults with transposition of the great arteries late after Mustard or Senning correction. Congenit Heart Dis. 2017; 12 (4): 448-457.

16. Lorenz Ch H, Walker E, Graham TP, Powers TA. Right Ventricular Performance and Mass by Use of Cine MRI Late After Atrial Repair of Transposition of the Great Arteries. Circulation 1995; 92 (9): 233-239.

17. R. Buys, Van De Bruaene A, Budts W, Delecluse C, Vanhees L. In adults with atrial switch operation for transposition of the great arteries low physical activity relates to reduced exercise capacity and decreased perceived physical functioning. Acta Cardiol 2012; 67 (1): 49-57.

18. Eicken A, Michell J, Hager A et al. Reduced Reserve to Stress after Atrial Baffle Repair for Transposition of the Great Arteries is Explained by Limited Ventricular Preload. Abstract 188. Circulation. 2007; 116: II 462.

19. Beerman LB, Neches WH, Fricker FJ, Mathews RA, Fischer DR, Park SC, Lenox CC, Zuberbuhler JR. Arrhythmias in transposition of the great arteries after the Mustard operation. Am J Cardiol. 1983; 51 (9): 1530-1534.

20. Janousek J, Paul T, Luhmer I, Wilken M, Hruda J, Kallfelz HC. Atrial baffle procedures for complete transposition of the great arteries: natural course of sinus node dysfunction and risk factors for dysrhythmias and sudden death. Z Kardiol 1994; 83 (12): 933-938.
21. Inuzuka R, Diller G P, Borgia F et al. Comprehensive Use of Cardiopulmonary Exercise Testing Identifies Adults with Congenital Heart Disease at Increased Mortality Risk in the Medium Term. Circulation 2012; 125 (2): 250-259.

22. Tomek V, Radvansky J, Adla T et al. Hemodynamics and Exercise Tolerance after Senning Operation for Transposition of Great Arteries and Its Limiting Factors: A Longitudinal Study. J Clin Exp Cardiolog 2016; 7 (4): 437-445.

23. Bobik L, Kovacikova L, Zahorec M, Danova K. Preoperative NTproBNP values in patients with atrioventricular septal defect and its role as a predictor of early postoperative course. Bratislava Medical Journal 2015; 116 (11): 648-653.

24. Chaix MA, Khairy P. Dextro-transposition of the great arteries: switching the switch. Transl Pediatr. 2019; 8 (5): 458-461.

25. Carazo M, Andrade L, Kim Y, Wilson W, Wu FM. Assessment and management of heart failure in the systemic right ventricle. Heart Fail Rev 2020. (Epub ahead of print)

26. Samarai D, Ingemansson SL, Gustafsson R, Thilén U, Hlebowicz J. Global longitudinal strain correlates to systemic right ventricular function. Cardiovasc Ultrasound. 2020; 18 (1): 4.

27. Schneider M, Beichl M, Nietsche C et al. Systematic Evaluation of Systemic Right Ventricular Function. Clin Med. 2019; 9 (1): 107-118.

28. Gavotto A, Abassi H, Rola $M$ et al. Factors associated with exercise capacity in patients with a systemic right ventricle. Int J Cardiol. 2019; 292 (8): 230-236. 